\title{
Motion Deblurring as Optimisation
}

\author{
V.S.Rao Veeravasarapu \\ Center for Visual Information Technology \\ International Institute of Information Technology \\ Hyderabad,India \\ vsrao@students.iiit.ac.in
}

\author{
Jayanthi Sivaswamy \\ Center for Visual Information Technology \\ International Institute of Information Technology \\ Hyderabad,India \\ jsivaswamy@iiit.ac.in
}

\begin{abstract}
Motion blur is one of the most common causes of image degradation. It is of increasing interest due to the deep penetration of digital cameras into consumer applications. In this paper, we start with a hypothesis that there is sufficient information within a blurred image and approach the deblurring problem as an optimisation process where the deblurring is to be done by satisfying a set of conditions. These conditions are derived from first principles underlying the degradation process assuming noise-free environments. We propose a novel but effective method for removing motion blur from a single blurred image via an iterative algorithm. The strength of this method is that it enables deblurring without resorting to estimation of the blur kernel or blur depth. The proposed iterative method has been tested on several images with different degrees of blur. The obtained results have been compared with state of the art techniques including those that require more than one input image. The results are consistently of high quality and comparable or superior to the existing methods which demonstrates the effectiveness of the proposed technique.
\end{abstract}

\section{Keywords}

Motion blurred image, De-blurring, Burg's entropy, Shannon's entropy, Univariate optimization.

\section{INTRODUCTION}

Many types of distortions limit the quality of digital images during image acquisition. Very often images are corrupted by motion blur. With the increased popularity of digital cameras for personal use, there simply is not enough knowledge or time to avoid using a long shutter speed, and the inevitable result is that captured images are blurred resulting in disappointment. Recovering un-blurred image from a single motion blurred image has long been a fundamental research problem in digital imaging. The standard

${ }^{*}$ Corresponding author

Permission to make digital or hard copies of all or part of this work for personal or classroom use is granted without fee provided that copies are not made or distributed for profit or commercial advantage and that copies bear this notice and the full citation on the first page. To copy otherwise, to republish, to post on servers or to redistribute to lists, requires prior specific permission and/or a fee.

ICVGIP'10, December 12-15, 2010, Chennai, India

Copyright 2010 ACM 978-1-4503-0060-5/10/12 ...\$10.00. way to express the relationship between the observed image $g(i, j)$ and its uncorrupted version $f(i, j)$ in noise-free environments is

$$
g(i, j)=f(i, j) * h(i, j)
$$

where $\mathrm{h}$ is the blur kernel or point spread function (PSF) and * is the convolution operator. Numerous methods have been proposed in the past for motion de-blurring. If one assumes that the blur kernel is shift-invariant, the problem reduces to that of image de-convolution. Image de-convolution can be further separated into the blind and non-blind cases. In non-blind de-convolution, the motion blur kernel is assumed to be known or computed elsewhere; the only task remaining is to estimate the un-blurred latent image. Traditional methods such as Weiner filtering and Richardson-Lucy (RL) de-convolution [11] were proposed decades ago, but continue to be widely used in many image restoration tasks because they are simple and efficient. However, these methods tend to suffer from unpleasant ringing artifacts that appear near strong edges. In the case of blind de-convolution [5] [6], the problem is even more ill-posed, since both the blur kernel and latent image are assumed unknown. The complexity of natural image structures and diversity of blur kernel shapes make it easy to over- or under-fit probabilistic priors [5].

In this paper, we begin our investigation of the blind deconvolution problem by exploring the major causes of visual artifacts such as ringing. Our study shows that the performance of current de-convolution methods is highly dependent on accurate estimation of motion blur parameters. We therefore observe that a better model of de-blurring and a more explicit handling of visual artifacts caused by the blur kernel estimate errors should substantially improve results. Based on these ideas, we propose an approach in which deblurring is achieved iteratively without explicitly estimating the blur kernel, by satisfying a set of conditions.

\section{RELATED WORK}

We first review techniques for non-blind de-convolution, where the blur kernel is known and only a latent image must be recovered from the observed, blurred image. The most common technique is the RL technique for de-convolution [11], which computes the latent image with the assumption that its pixel intensities conform to a Poisson distribution. Donatelli et al. [4] use a PDE-based model to recover a latent image with reduced ringing by incorporating an anti-reflective boundary condition and a re-blurring step. A common approach in the signal processing community to the de-convolution problem is to transpose the 
problem to the wavelet or the frequency domain (an example is [15]); However, many of these papers lack experiments in de-blurring real photographs, and few of them attempt to model error in the estimated kernel. Levin et al. [8] use a sparse derivative prior to avoid ringing artifacts in de-convolution. Most non-blind de-convolution methods assume that the blur kernel contains no errors, however, even small kernel errors can lead to significant artifacts. Finally, many of these de-convolution methods require complex parameter settings and long computation times.

Blind de-convolution is a significantly more challenging and ill-posed problem, since the blur kernel is also unknown. Some techniques make the problem more tractable by leveraging additional input, such as multiple images. Rav-Acha et al. [18] utilise the information in two motion blurred images, while Yuan et al. [22] use a pair of images, one blurred and one noisy, to facilitate capture in low light conditions. Another strategy adopted has been to take advantage of additional, specialized hardware. Ben-Ezra and Nayar [2] attach a low-resolution video camera to a high-resolution still camera to help in recording the blur kernel. Raskar et al. [17] flutter the opening and closing of the camera shutter during exposure to minimize the loss of high spatial frequencies. This method requires the object motion path to be specified by the user. The most ill-posed problem is single-image blind de-convolution, which must both estimate the PSF and the latent image. Early approaches usually assume simple parametric models for the PSF such as a low-pass filter in the frequency domain [7] or a sum of normal distributions [9]. Fergus et al.[5] showed that blur kernels are often complex and sharp; they use ensemble learning (Miskin and MacKay [12]) to recover a blur kernel while assuming a some statistical distribution for natural image gradients. A variational method is used to approximate the posterior distribution and the RL technique is used for de-convolution. Jia et al. [6] recovered the PSF from the perspective of transparency by assuming the transparency map of a clear foreground object should be two-tone. This method is limited by a need to find regions that produce high quality matting results. Qi shan et al. [20] creates an unified probabilistic framework for both blur kernel estimation and latent image recovery by allowing these two estimation problems to interact to avoid local minima and ringing artifacts.

Our hypothesis is that there is sufficient information in the blurred image to aid deblurring process. Accordingly we aim to devise a solution which takes a novel different approach to the problem. We first present the necessary basics and then present the proposed method.

\section{MODELING MOTION BLUR}

Let us assume that a linear, non-recursive (FIR) model represents the degradation of digital (sampled) images caused by motion blur. The original, blur free $M \times N$ image $f$ is convolved with a blur kernel $h$. De-blurring images requires the application of the de-blurring operator $D$, which produces a de-blurred image $f * h$ when applied to the blurred image $g$, that is $D(g)=f * h$.

The blur kernel provides information of the underlying motion during the capture process. In the most simple case, such as for a uniform linear motion along the $\mathrm{x}$-axis with a speed of $\mathrm{k}$ pixels during the capturing period, the PSF is given by a one-dimensional vector of the length $\mathrm{k}+1$ :

$$
h_{\text {lin }}=\frac{1}{k+1}[111 \ldots 1]
$$

In [2] propose a method to determine the motion paths during the capturing process. Their analysis shows that the model for the PSF has to be extended to represent motion in a two-dimensional plane. The PSF is a matrix $\mathrm{h}$ of size $U \times V$, where each entry $h(i, j) \mathrm{i}=1,2, \ldots, U, \mathrm{j}=1,2, \ldots, V$ represents the percentage the camera has been displaced by $i-(U / 2), j-(V / 2)$ from the centre during the capture.

$$
h=\frac{1}{K}\left[\begin{array}{cccc}
h_{1,1} & h_{1,2} & \ldots & h_{1, V} \\
h_{2,1} & h_{2,2} & \ldots & \\
\vdots & \vdots & \ddots & \vdots \\
h_{U, 1} & h_{U, 2} & \ldots & h_{U, V}
\end{array}\right]
$$

Where the parameter $\mathrm{K}$ is a normalizing constant to ensure that the sum over the entries of the matrix equals to 1 . The rest of the paper is organized as follows. In Section 4, the details of our method are introduced. Experimental results and comparisons are provided in Section 5. And finally we present the conclusion in Section 6 .

\section{PROPOSED METHOD}

The proposed method consists of two parts. i) direction detection to estimate the direction of motion $(\phi)$ and ii) compensation for blur. These are presented in detail below.

\subsection{Direction Detection}

Since motion blur is essentially directional averaging, it results in parallel white bands in the Fourier spectrum of a degraded image. This has been used to effectively determine the direction $(\phi)$ of the motion blur [13] [14]. We extract the blur direction using the same principle but using the Radon transform: Let $|G(u, v)|$ be the amplitude spectrum of the given blurred image $g[m, n]$. We take the Radon transform (RT) of this function $|G(u, v)|$ to find the direction of these bands and find the angle corresponding to the maxima in the RT. After finding the motion direction estimation, the blurred image $\mathrm{g}$ is rotated to align it with the computed motion direction. The desired deblurred image is estimated by compensating for the blur as described next.

\subsection{Compensation}

Deblurring can be viewed as a problem where a set of corrupted data (blurred pixel values) is given and the process of deblurring has to recover the original pixel values while satisfying some conditions. This leads to casting the compensation step as an optimisation process which satisfies a set of conditions. The requisite conditions can be identified from the basic principles underlying the blur process.

\subsubsection{C1. Conservation of Mass}

If the blur kernel is a normalized one, the mean value of the signal will not change after convolution. Given that the blur kernel in eq 3 is normalized, this implies the sum of all pixel values in the blurred image must equal to that in the restored image [3]. The sum of all pixel values in blurred image as $M_{1}$ is given as

$$
M_{1}=\sum \sum g(i, j)
$$




\subsubsection{C2. Conservation of Energy}

The degradation process obeys Law of conservation of energy as the motion of an object or of the camera does not need any optical energy [3]. Hence, the energy of a blurred image is same as that in the original image. This energy denoted by $M_{2}$ is

$$
M_{2}=\sum \sum g(i, j)^{2}
$$

\subsubsection{C3. Entropy condition}

Many restoration algorithms are based on minimization of Shannons entropy E (examples are [3],[16]), which is given as

$$
E=-\sum \sum g(i, j) \log [g(i, j)]
$$

The basic assumption behind these methods is that the Shannons entropy of the original image is less than that of the degraded image. This may not hold for very large-size blur kernels. We have found that the entropy of images increases with blur depth up to a certain level, after which it starts decreasing. Hence, we include the next condition.

\subsubsection{C4. Information condition}

For a related inversion problem in speech processing, an alternate measure for entropy, namely the Burg entropy is used which is defined as

$$
B=-\sum \sum \log [g(i, j)]
$$

Burg's entropy has been argued to be a better representation of information content and has previously been used in image reconstruction [1]. In the context of restoration, it has been shown that $B$ value of a restored image is higher than that of the corrupted source image [16]. In the proposed method, this entropy measure is used and deblurring aims to maximise the same.

\subsubsection{Compensate Function}

Given a current pixel value in a motion blurred image, its value is likely to be due to an averaging process over its immediate neighbours. Hence, a compensate function $C_{f}$ is defined to reverse this process. The function for two adjacent pixels is defined as follows:

$$
C_{f}^{2}(i, j)=a . g(i, j)-b . g(i, j-1)-c \cdot g(i, j+1)
$$

The function for four adjacent pixels is defined as

$$
\begin{array}{r}
C_{f}^{4}(i, j)=a . g(i, j)-b . g(i, j-1)-c . g(i, j+1) \\
-d . g(i, j-2)-e . g(i, j+2)
\end{array}
$$

where a, b, c, d and e are unknown re-weight parameters which will be found iteratively. An illustration for processing a row of pixels is shown in Figure 1.

From the Figure 1, it can be seen that estimation of a current pixel depends on 3 pixels from the previous iteration. Hence, after $\mathrm{k}$ iterations, estimation of a current pixel depends on $3 \mathrm{k}$ pixels in the input blurred image. So the number required iterations is indirectly based on the length of blur $(L)$. In each iteration, the optimum values of of the re-weight parameters are estimated by imposing the condition set $\mathrm{C} 1$ through $\mathrm{C} 4$. Next, we present an algorithm for the same. For simplicity we assume a $C_{f}^{2}$ case.

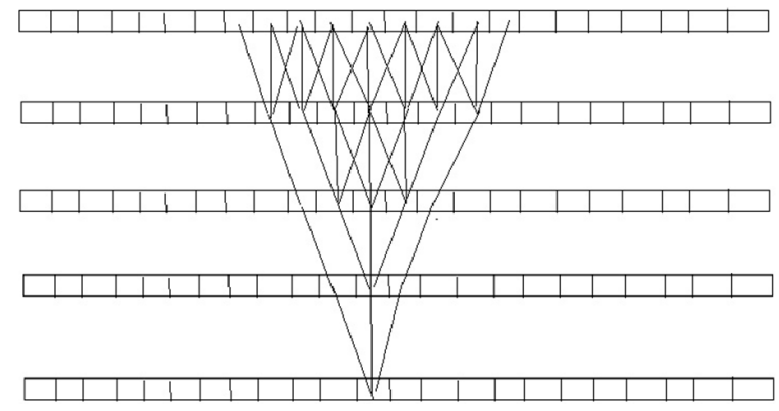

Figure 1: Four iterations of a row in an image using $C_{f}^{2}$.

\subsubsection{Algorithm for Optimization}

The problem at hand is optimization of weight parameters $\mathrm{a}, \mathrm{b}$, and $\mathrm{c}$ with respect to the condition set. The uni-variate method is adopted for a solution of this problem, by multiplying the step size $S_{i}$ by very small increment $\varepsilon$. In this method, only one parameter is changed at a time to produce a sequence of improved approximations to reach the optimum point. Starting at a base point $P_{i}=(a, b, c)_{i}$ in the $i^{\text {th }}$ iteration, the value of any one of $(n-1)$ parameters is fixed while others are varied. The purpose is to produce a new base point $P_{i+1}$. The search is now continued in a new direction. The new direction is obtained by changing any one of the $n-1$ parameters that has been fixed in the previous iteration. After all the $n$ directions are searched sequentially, the first cycle is completed and values of $a, b$ and $\mathrm{c}$ are obtained. These are placed in a dummy image which forms the input for the next iteration. The entire process of sequential optimization is repeated until the values of $(\mathrm{a}, \mathrm{b}, \mathrm{c})$ is approximately $(1,0,0)$. The choice of the direction and the step length in the modified uni-variate method is summarized here.

\section{Modified Univariate Algorithm}

1. Choose a starting point $P_{i}=(a, b, c)_{i}$ and set $\mathrm{i}=1$.

2. Find the search direction $S_{i}$ as 8

$$
\mathrm{S}_{i}^{T}= \begin{cases}((1,0,0,0,0, \ldots) & \mathrm{i}=1, \mathrm{n}+1,2 \mathrm{n}+1 \\ (0,1,0,0,0, \ldots) & \mathrm{i}=2, \mathrm{n}+2,2 \mathrm{n}+2 \\ \vdots & \\ (0,0,0,0,0, \ldots, 1) & \mathrm{i}=\mathrm{n}, 2 \mathrm{n}, 3 \mathrm{n}, \ldots\end{cases}
$$

3. For the current direction $S_{i}$, find the values of $M_{1}$, $M_{2}, \mathrm{E}$ and $\mathrm{B}$ and check if condition set is satisfied. If condition set is not satisfied, find whether the entropy (E) values decreases in the positive or negative direction. For this, we take a small probe length $(\varepsilon)$, also called learning factor and evaluate $E_{i}=E\left(P_{i}\right)$, $E_{i}^{+}=E\left(P_{i}+\varepsilon S_{i}\right)$ and $E_{i}-=E\left(P_{i}-\varepsilon S_{i}\right)$. If $E_{i}^{+}>E_{i}^{-}$ , $S_{i}$ will be the correct direction for decreasing the values of $E_{i}$, and if $E_{i}^{+}<E_{i}^{-},-S_{i}$ will be the correct direction. If both $E_{i}^{+}$and $E_{i}^{-}$are less than $E_{i}$, we take $P_{i}$ as the minimum of the two.

4. Set $P_{i+1}=P_{i}+\varepsilon S_{i}$.

5. $E_{i}+1=E\left(P_{i+1}\right)$.

6. Set $i=i+1$ and go to step 2 . Continue this procedure until $(\mathrm{a}, \mathrm{b}, \mathrm{c})$ satisfies the condition set. 

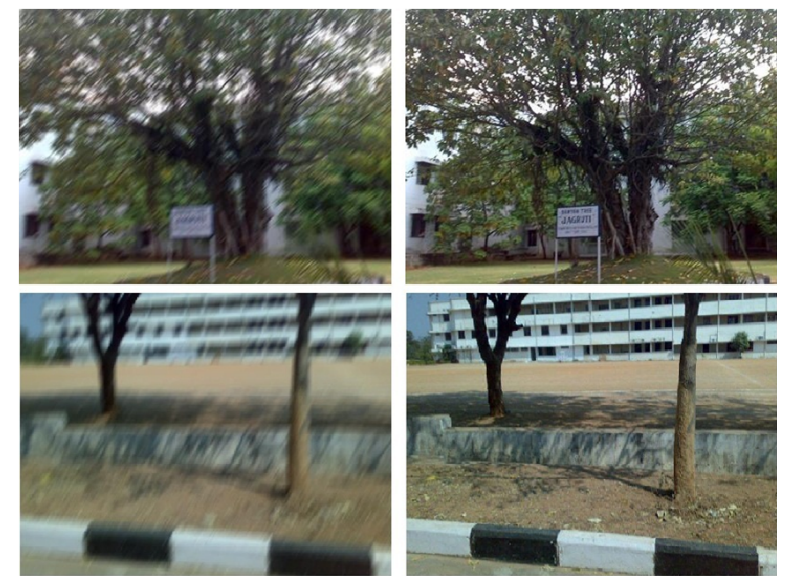

Figure 2: (a) First column: blurred images captured by a hand-held camera. (b)second column: corresponding outputs of our method.

We have taken a unit step length for computational simplicity. The algorithm for the de-blurring technique is as follows.

Algorithm for Iterative Motion deblurring (IMD)

1. Find the angle of direction of motion $(\phi)$

2. Rotate the coordinate system by an angle $\phi$.

3. Apply the compensate function to rotated R, G, and B planes of blurred image individually.

4. Impose the condition set using Modified Uni-variate method for each plane.

5. Create dummy image planes with a,b, and c.

6. Repeat 3 to 6 steps with these dummy image planes until we get $a=1, b=0, c=0$ approximately for each plane.

7. Anti-rotate the image.

8. Display the restored image.

Any algorithm that performs de-convolution in the Fourier domain needs a post processing step to suppress ringing artifacts at the image boundaries; for example, Fergus et al. [5] process the image near boundaries using the Matlab edgetaper command. We instead use the approach of Liu and Jia [10] to suppress the ringing. Some results of this method are provided in Figure 2.

\section{EXPERIMENTAL RESULTS}

The proposed iterative deblurring algorithm was tested on numerous images. We present some sample results in this section. Two blurred test images captured using a handheld camera and the corresponding deblurred results obtained by the proposed method is shown in Figure 2.

In order to assess the performance of to proposed method against existing methods a set of comparisons were carried out: Deblurring i) without use of additional images and ii) with use of additional information/images. Henceforth, the proposed technique is referred to as IMD for convenience.

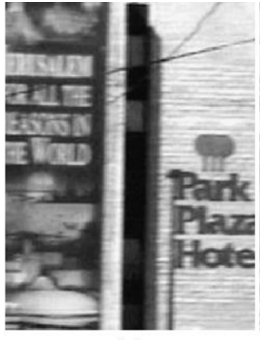

(a)

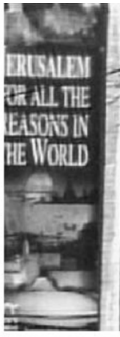

(b)

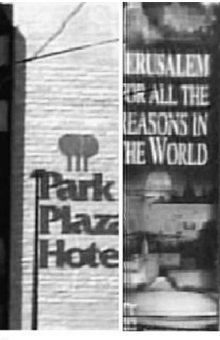

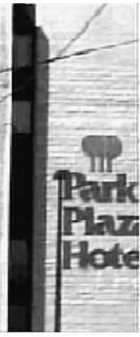

(c)
Figure 3: (a) motion blurred image used in [18]. (b) Deblurred result from [18] using information from two blurred images. (c) IMD result using only blurred image shown in (a).

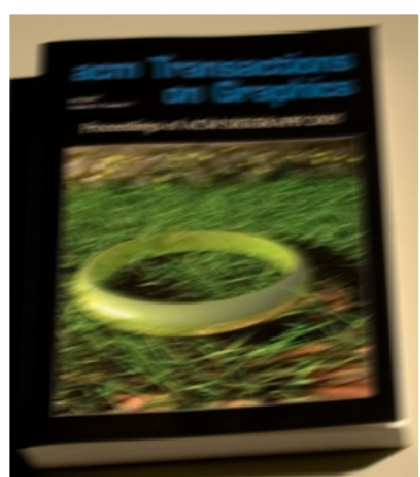

(a)

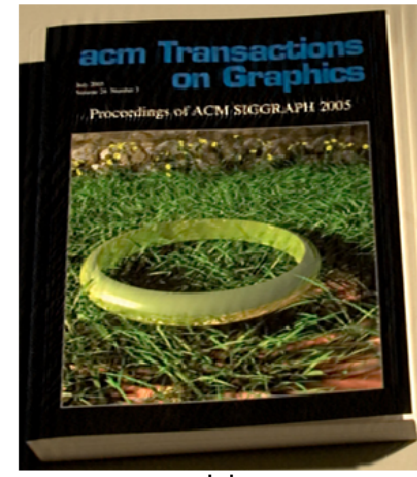

(c)

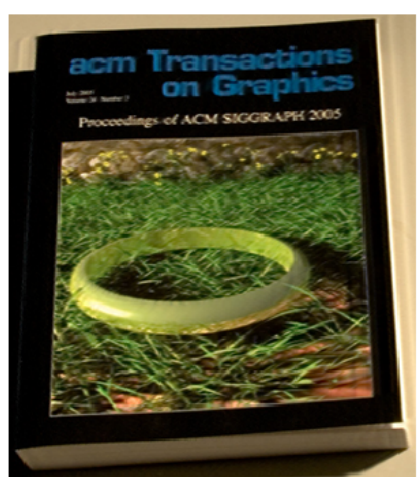

(b)

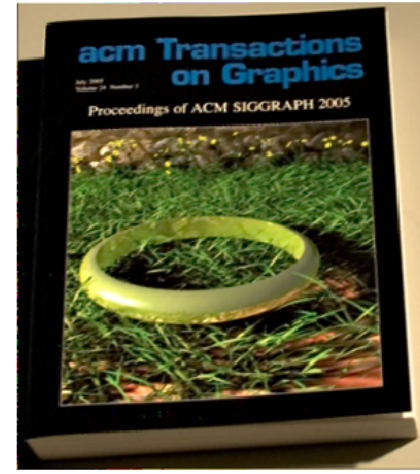

(d)
Figure 4: Non-blind de-convolution example. (a) blurred image used in [20]. Deblurred results of (b) RL algorithm (c) sparse prior method [8] and (d) IMD.

\section{Deblurring without use of additional images}

A uniformly blurred image and the deblurred results are shown in Figure. 4(a). The results of RL, Levin et al. [8] and IMD techniques are shown in Figure 4 (b), (c) respectively. IMD result exhibits sharper image details and fewer artifacts such as ringing around sharp edges, than the others.

We next illustrate blind de-convolution on two test images taken from [20]; These are shown in Figure 5 and Figure 6. The blur is due to camera shake. The results of two sample techniques namely [5] and [6] which are based on the RL technique are also taken from [20]. The degree of blur in the second image shown in Figure 6 (a)) is caused by a large-size 
kernel, which is challenging for kernel estimation. The results of IMD is shown alongside for comparison for both test images. The IMD results for the green toy image is comparable with some areas such as the right ear, being restored better. The colour and sheen are superior in the result of [6]. The IMD results for the second test image in Figure 6 (a)) is in comparison clearer compared to the other two techniques. This implies that IMD is superior at handling high degree of blur. A comparison with the most recent deblurring method [20] which uses a probabilistic approach is shown in Figure 7. The two results appear to be of similar quality.

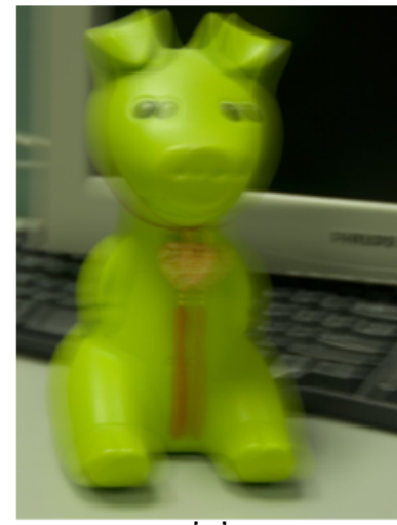

(a)

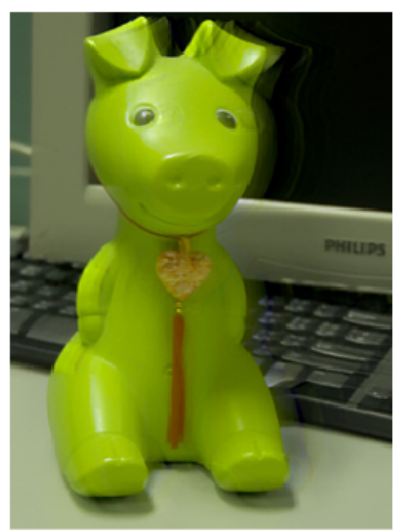

(c)

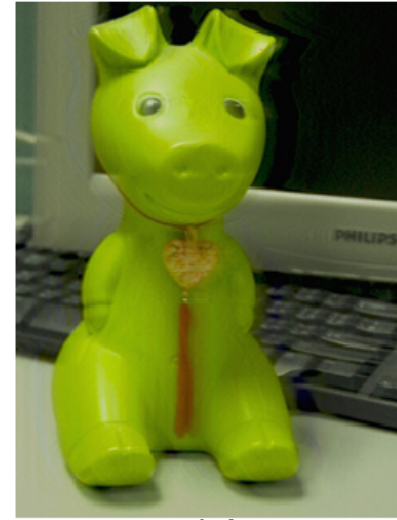

(b)

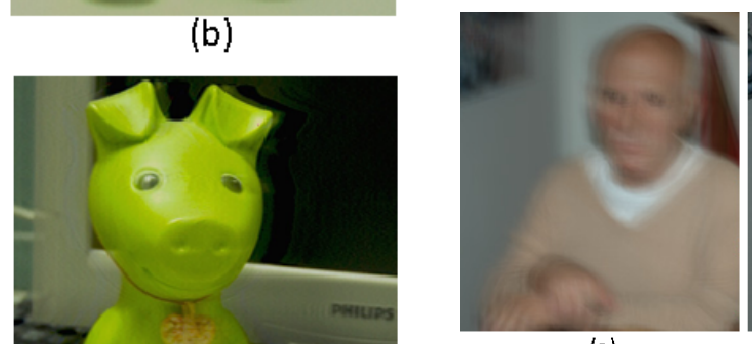

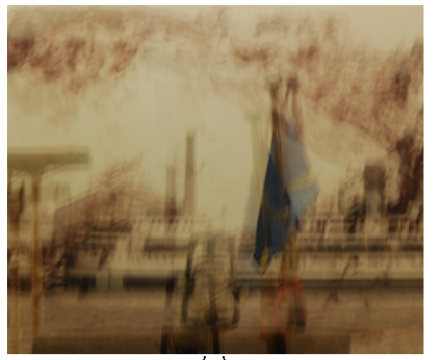

(a)

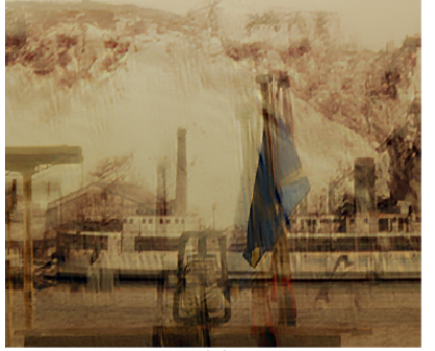

(c)

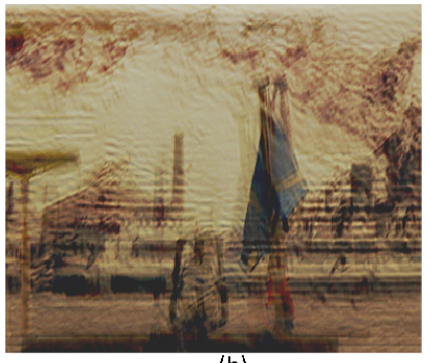

(b)

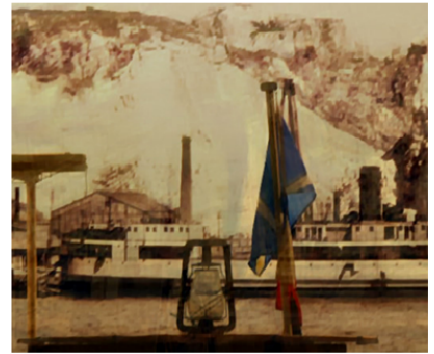

(d)
Figure 6: Blind deconvolution example 2. a) input blurred image; de-blurring results of b) Fergus et al. [5], c) Jia et al. [6] and d) IMD. Other two methods use RL de-convolution to restore the blurred image. (a)

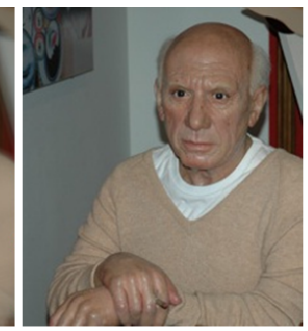

(b)

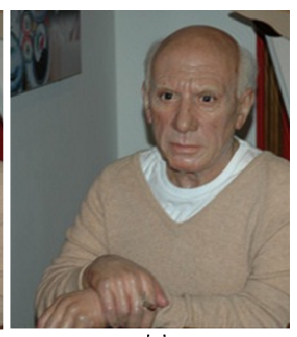

(c)
Figure 7: Blind deconvolution example 3. a) input image; de-blurring results of b) $[20]$, b) and c) IMD.

image.

Finally, two more challenging real examples and IMD results are shown in Figure 11, all containing complex structures and blur from a variety of camera motions. The ringing, even around strong edges and textures, are significantly reduced. The remaining artifact is caused mainly by the fact that the motion blur is not absolutely spatially invariant. Using a hand-held camera, slight camera rotation and motion parallax are easily introduced by Shan et al. [21] .

\section{CONCLUSION AND DISCUSSION}

In this paper, a novel image restoration method has been proposed to remove camera motion blur from a single image by viewing deblurring as an optimisation process. The method does not involve estimation of the blur kernel or blur depth and achieves the deblurring iteratively. Our main contributions are an effective model for removing blur that accounts for its spatial distribution, and a local prior to suppress ringing artifacts. This model improves unblurred image estimation even with a very simple compensate function after a modified uni-variate optimization process is applied. 


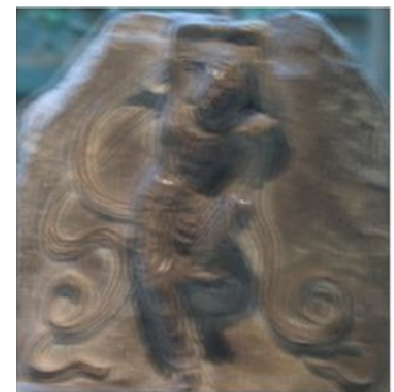

(a)

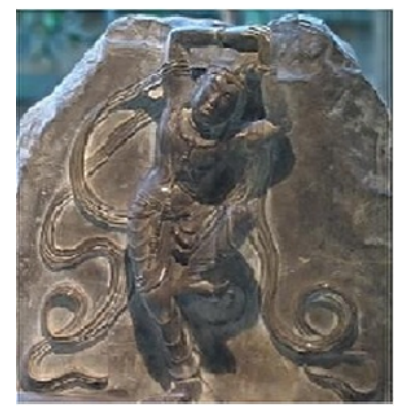

(c)

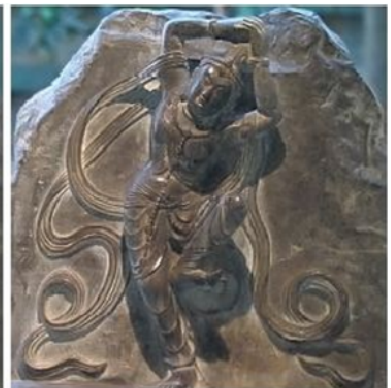

(b)
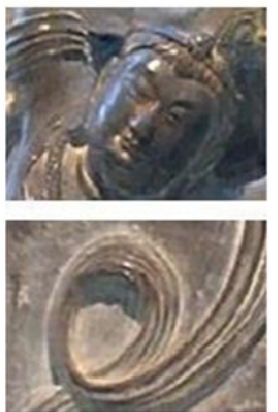

(d)
Figure 8: Deblurring with additional input images from [22]. a) The blurred input image, b) result from [22], c) IMD result with only blurred image as input and d) some close-ups of our results.

The proposed technique avoids the computation of blur

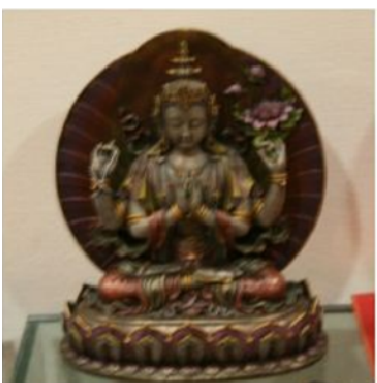

(a)

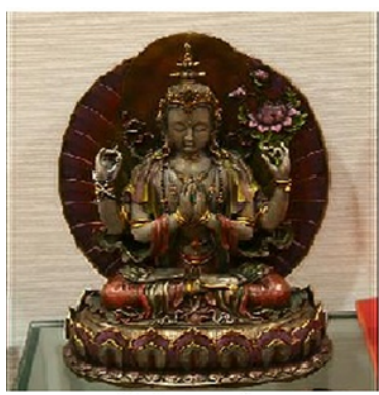

(c)

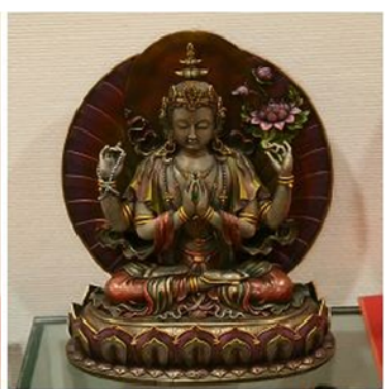

(b)

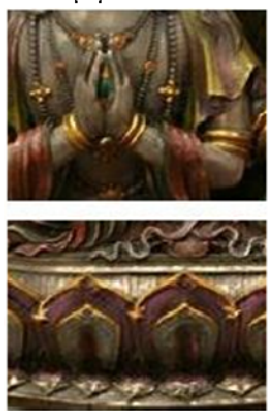

(d)
Figure 9: Deblurring with additional input images from [22]. a) The blurred input image, b) result from $[22]$, c) IMD result with only blurred image as input and d) some close-ups of our results. depth parameter which is often erroneous. The successful results obtained with this technique is principally due to the optimization scheme that re-weights the relative membership values of neighboring pixels in current pixel value, over the course of the optimization. We have found that this re-weighting approach can work very accurately in case of horizontal uniform motion blur even if it is blurred by a large-size kernel.

The proposed technique was found to successfully deblur most motion blurred images. However, one failure mode occurs when the blurred image is affected by blur that is not shift-invariant, e.g., from slight camera rotation or nonuniform object motion. An interesting direction of future work is to explore the removal of non-shift-invariant blur using a general compensate function assumption.

Another interesting observation that arises from our work is that images, which are blurred with a very large-size kernel, contain more information than the original images. Our results show that for moderately blurred images, edge, color, and texture information can be satisfactorily recovered. A

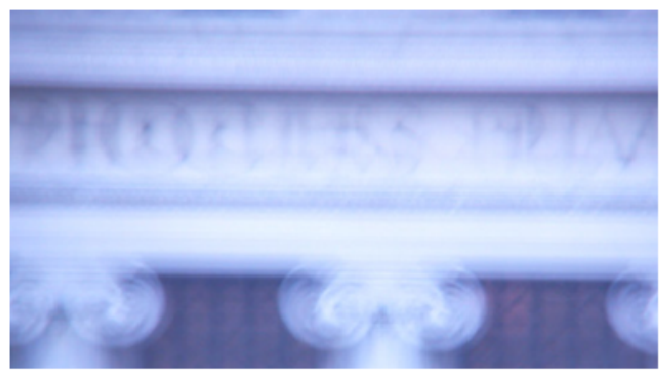

(a)

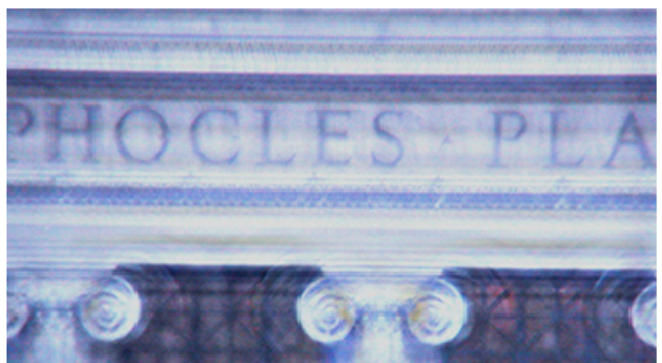

(b)

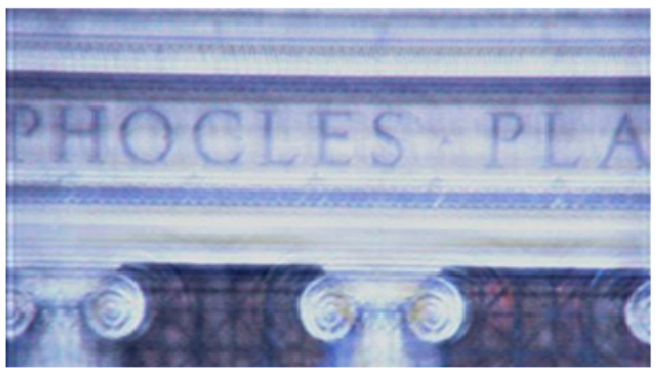

(c)

Figure 10: Deblurring with additional input images from [2] a) a motion blurred image of a building from the paper of Ben-Ezra and Nayar[2], b) their result using information from an attached video camera to estimate camera motion and c) IMD result obtained with one input image. 

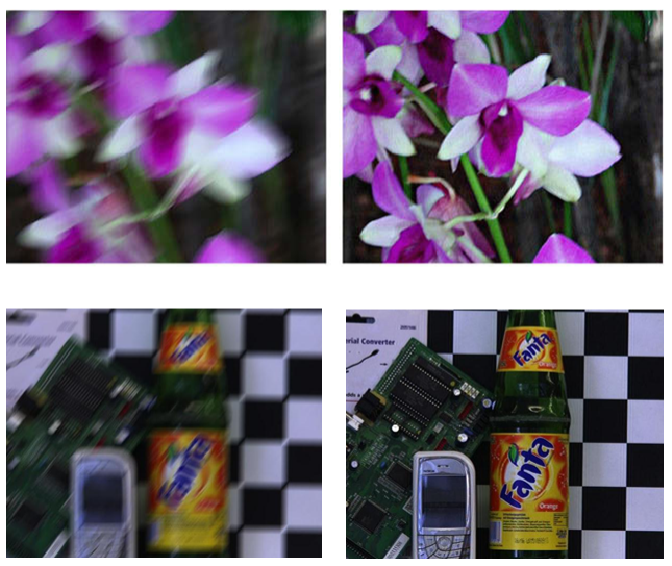

Figure 11: Deblurring on two challenging cases.(a) the captured blurred images from [19] (b) IMD results.

successful motion de-blurring method, thus, makes it possible to take advantage of information that is currently buried in blurred images, which may find applications in many imaging-related tasks, such as image understanding, 3D reconstruction, and video editing.

\section{REFERENCES}

[1] C. Auyeung and R.M.Mersereau. A dual approach to signal restoration. In Information Sciences: Springer Series, 23:21-56, 1991.

[2] M. Ben-Ezra and S. Nayar. Motion-based motion deblurring. IEEE Transactions on Pattern Analysis and Machine Intelligence, 26(6):689-699, 2004.

[3] D. Cunningham. Image motion deblurring. Online Archive, 2006.

[4] M. Donatelli, C. Estatico, A. Martinelli, and S. Serracapizzano. Improved image deblurring with antireflective boundary conditions and re-blurring. Inverse Problems, 22(6):2035-2053, 2006.

[5] R. Fergus, B. Singh, A. Hertzmann, S. T. Roweis, and W. Freeman. Removing camera shake from a single photograph. Acm Transactions On Graphics, 25:787-794, 2006.

[6] J. Jia. Single image motion deblurring using transparency. Proc. CVPR, 2007.

[7] S. K. Kim and J. Paik. Out-of-focus blur estimation and restoration for digital auto-focusing system. Electronics Letters, 34(12):1217-1219, 1998.

[8] A. Levin, R. Fergus, F. Durand, and B. Freeman. Image and depth from a conventional camera with a coded aperture. Proc. Siggraph, 2007.

[9] A. Likas and N. A. Galatsanos. Variational approach for bayesian blind image deconvolution. IEEE Trans. om Signal Processing, 52(8):2222-2233, 2004.

[10] R. Liu and J. Jia. Reducing boundary artifacts in image deconvolution. Proc. ICIP, 2008.

[11] L. Lucy. Bayesian-based iterative method of image restoration. Journal of Ast., 1974.

[12] J. Miskin and D. Mackay. Ensemble learning for blind image separation and deconvolution. Advances In Independent Component Analysis, pages 123-141,
2000.

[13] M. E. Moghaddam and M. Jamzad. Finding point spread function of motion blur using radon transform and modelling the motion length. Proc. ISSPIT, pages 314-317, 2004.

[14] M. E. Moghaddam and M. Jamzad. Linear motion blur parameter estimation in noisy images using fuzzy sets and power spectrum. EURASIP Journal on Advances in Signal Processing, 2006.

[15] R. Neelamani, H. Choi, and R. G. Baraniuk. Fourier-wavelet regularized deconvolution for illconditioned systems. IEEE Transactions On Signal Processing, 52:418-433, 2004.

[16] D. noll. Restoration of degraded images with maximum entropy. Journal of Global Optimization, 10:91-103, 1997.

[17] R. Raskar, A. Agrawal, and J. Tumblin. Coded exposure photography: Motion deblurring using fluttered shutter. ACM Transactions on Graphics, 25(3):795-804, 2006.

[18] A. Rav-Acha and S. Peleg. Two motion blurred images are better than one. Pattern Recognition Letters, 26:311-317, 2005.

[19] S. Schuon and K. Diepold. Comparison of motion deblur algorithms and real world deployment. IAC-2007, B1:1-11, 2007.

[20] Q. Shan, J. Jia, and A. Agarwala. High-quality motion deblurring from a single image. Proc. SIGGRAPH, 2009.

[21] Q. Shan, W. Xiong, and J. Jia. Rotational motion deblurring of a rigid object from a single image. Proc. ICCV, 2007.

[22] L. Yuan, J. Sun, L. Quan, and H. Y. Shum. Image deblurring with blurred/noisy image pairs. Proc. Siggraph, 2007. 